\title{
Kingdom of the Netherlands-Aruba: Statistical Appendix
}

This statistical appendix on the Kingdom of the Netherlands-Aruba was prepared by a staff team of the International Monetary Fund as background documentation for the periodic consultation with the member country. It is based on the information available at the time it was completed on October 6,2010. The views expressed in this document are those of the staff team and do not necessarily reflect the views of the government of Aruba or the Executive Board of the IMF.

The policy of publication of staff reports and other documents by the IMF allows for the deletion of market-sensitive information.

\author{
Copies of this report are available to the public from \\ International Monetary Fund • Publication Services \\ $70019^{\text {th }}$ Street, N.W. • Washington, D.C. 20431 \\ Telephone: (202) 623-7430 • Telefax: (202) 623-7201 \\ E-mail: publications@imf.org Internet: http://www.imf.org
}

\section{International Monetary Fund Washington, D.C.}




\section{INTERNATIONAL MONETARY FUND \\ KINGDOM OF THE NETHERLANDS—ARUBA}

\section{Statistical Appendix}

Prepared by Johannes Wiegand and Vladimir Munteanu

Approved by the European Department

October 6, 2010

Contents

Page

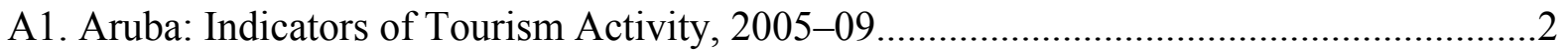

A2. Aruba: Components of GDP, 2005-09 ...................................................................2

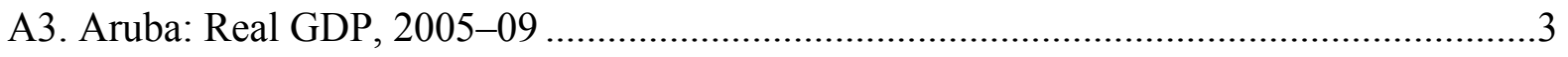

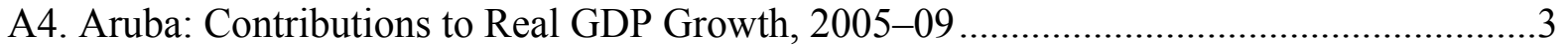

A5. Aruba: Changes in Consumer Price Index, 2006-09 .......................................................4

A6. Aruba: Legal Minimum Wages, 2006-09.................................................................4

A7. Aruba: Operations of the Central Government, 2006-09 ...........................................5

A8. Aruba: Operational Budget of the Social Insurance Bank (SVB), 2005-09 ...................6

A9. Aruba: Financial Balance of AZV, 2006-09 ................................................................. 7

A10. Aruba: Balance of Payments Summary, 2005-09 .................................................... 7

A11. Aruba: Balance of Payments Summary, 2005-09 ....................................................8

A12. Aruba: Non-Oil Balance of Payments Summary, 2005-09 ........................................

A13. Aruba: Non-Oil Balance of Payments Summary, 2005-09 ........................................9

A14. Aruba: Bank-Like Institutions_-Balance Sheet, 2005-09 ..........................................10

A15. Aruba: Life Insurance Companies_-Balance Sheet, 2005-08....................................10

A16. Aruba: Pension Funds_-Balance Sheet, 2005-09 ......................................................11

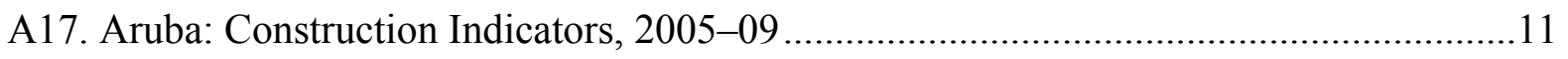

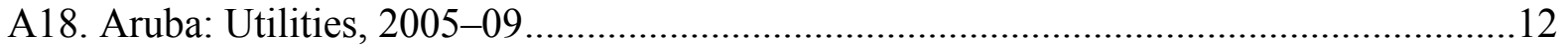

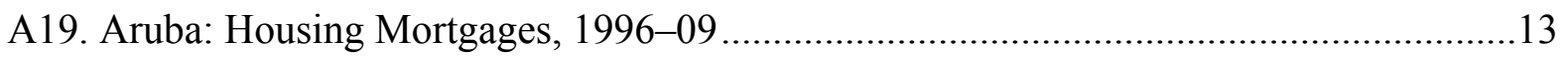


Table A1. Aruba: Indicators of Tourism Activity, 2005-09

(Millions of Aruban florins at current prices; unless otherwise indicated)

\begin{tabular}{lrrrrr}
\hline & 2005 & 2006 & 2007 & 2008 & 2009 \\
\hline Total revenue & 1,953 & 1,926 & 2,244 & 2,523 & 2,320 \\
$\quad$ (percent change) & 3.7 & -1.4 & 16.5 & 12.5 & -8.1 \\
(percent change, in real terms) & 0.3 & -4.8 & 16.5 & 12.5 & -8.1 \\
& & & & & \\
Stay-over visitors (thousands) & 733 & 694 & 772 & 827 & 813 \\
$\quad$ (percent change) & 0.6 & -5.2 & 11.2 & 7.1 & -1.7 \\
$\quad$ Tourist nights (thousands) & 5,695 & 5,471 & 5,880 & 6,265 & 6,173 \\
$\quad$ percent change) & 1.0 & -3.9 & 7.5 & 6.5 & -1.5 \\
Average stay (nights) & 7.8 & 7.9 & 7.6 & 7.6 & 7.6 \\
Expenditure per tourist & & & & & \\
$\quad$ (Aruban florins) & 343 & 352 & 382 & 403 & 376 \\
Hotel occupancy rate (percent) & 83.9 & 74.3 & 74.5 & 73.2 & 72 \\
& & & & & \\
Cruise tourists (thousands) & 553 & 591 & 482 & 556 & 607 \\
$\quad$ (percent change) & -4.1 & 7.0 & -18.5 & 15.4 & 9.1 \\
\hline
\end{tabular}

Sources: CBA, Quarterly Bulletin; Aruba Tourism Authority; CBS; Aruba Hotel and Tourism Association; and the Cruise Tourism Authority.

Table A2. Aruba: Components of GDP, 2005-09 (Percent of GDP)

\begin{tabular}{lrrrrr}
\hline & 2005 & 2006 & 2007 & 2008 & 2009 \\
\hline Gross domestic product & 100.0 & 100.0 & 100.0 & 100.0 & 100.0 \\
Private absorption & 84.1 & 86.6 & 87.9 & 87.9 & 83.5 \\
$\quad$ Consumption & 52.9 & 53.6 & 55.9 & 55.9 & 55.9 \\
$\quad$ Private investment 1/ & 31.3 & 33.0 & 32.0 & 32.1 & 27.6 \\
Government absorption & 23.9 & 24.3 & 21.8 & 22.5 & 24.1 \\
$\quad$ Consumption & 22.4 & 22.8 & 20.6 & 20.9 & 22.3 \\
$\quad$ Investment 2/ & 1.5 & 1.5 & 1.2 & 1.6 & 1.8 \\
Net exports 3/ & -8.1 & -10.9 & -9.8 & -10.4 & -7.7 \\
$\quad$ Export of goods and services & 68.4 & 65.2 & 65.8 & 69.6 & 64.8 \\
$\quad$ Import of goods and services & 76.5 & 76.0 & 75.5 & 79.9 & 72.4 \\
\hline
\end{tabular}

Source: CBS.

1/ Private investment is equal to private gross fixed capital formation and private changes in inventories. The investment of the oil sector is included in these figures.

2/ Public investment is equal to public gross fixed capital formation and public changes in inventories.

3/ Exports less imports of goods and services. The activities of the oil refinery are registered on a net basis, namely a refining fee is computed and used as the oil sector's output and export. Other goods and/or services imported or exported by the oil sector are included in the trade figures. Refinery products deliver to Aruban users are registered as import. 
Table A3. Aruba: Real GDP, 2005-09

(Millions of Aruban florins at constant 1995 prices)

\begin{tabular}{lrrrrr}
\hline & 2005 & 2006 & 2007 & 2008 & 2009 \\
\hline Gross domestic product & 2,973 & 3,006 & 3,048 & 3,069 & 2,832 \\
(percent change) & 2.0 & 1.1 & 1.4 & 0.7 & -7.7 \\
Private absorption & 2,732 & 2,847 & 2,963 & 2,959 & 2,610 \\
Consumption & 1,633 & 1,665 & 1,773 & 1,743 & 1,645 \\
Private investment 1/ & 1,099 & 1,182 & 1,190 & 1,216 & 965 \\
Government absorption & 733 & 732 & 691 & 715 & 743 \\
Consumption & 678 & 678 & 645 & 656 & 682 \\
Investment 2/ & 54 & 54 & 46 & 59 & 61 \\
Net exports 3/ & -492 & -573 & -607 & -605 & -522 \\
Export of goods and services & 2,164 & 2,103 & 2,180 & 2,334 & 2,018 \\
Import of goods and services & 2,656 & 2,676 & 2,786 & 2,938 & 2,540 \\
\hline
\end{tabular}

Source: CBS.

1/ Private investment is equal to private gross fixed capital formation and private changes in inventories. The investment of the oil sector is included in these figures.

2/ Public investment is equal to public gross fixed capital formation and public changes in inventories.

3/ Exports less imports of goods and services. The activities of the oil refinery are registered on a net basis, namely a refining fee is computed and used as the oil sector's output and export. Other goods and/or services imported or exported by the oil sector are included in the trade figures. Refinery products delivered to Aruban users are registered as import.

Table A4. Aruba: Contributions to Real GDP Growth, 2005-09 (Percent)

\begin{tabular}{lrrrrr}
\hline & 2005 & 2006 & 2007 & 2008 & 2009 \\
\hline Private absorption & 8.3 & 3.8 & 3.9 & -0.1 & -11.4 \\
Consumption & 2.3 & 1.1 & 3.6 & -1.0 & -3.2 \\
Private investment 1/ & 6.0 & 2.8 & 0.3 & 0.8 & -8.2 \\
Government absorption & 0.8 & 0.0 & -1.3 & 0.8 & 0.9 \\
Consumption & 0.7 & 0.0 & -1.1 & 0.3 & 0.9 \\
Investment 2/ & 0.1 & 0.0 & -0.3 & 0.4 & 0.1 \\
Exports less imports 3/ & -7.0 & -2.7 & -1.1 & 0.1 & 2.7 \\
Goods & 3.4 & -2.1 & 2.6 & 5.1 & -10.3 \\
Services & 10.4 & 0.7 & 3.7 & 5.0 & -13.0 \\
Real GDP growth & 2.0 & 1.1 & 1.4 & 0.7 & -7.7 \\
Memorandum item: & & & & & \\
Nominal growth rate & 4.4 & 4.2 & 7.5 & 7.3 & -7.7 \\
\hline
\end{tabular}

1/ Private investment is equal to private gross fixed capital formation and private changes in inventories. The investment of the oil sector is included in these figures.

2/ Public investment is equal to public gross fixed capital formation and public changes in inventories.

3/ Exports less imports of goods and services. The activities of the oil refinery are registered on a net basis, namely a refining fee is computed and used as the oil sector's output and export. Other goods and/or services imported or exported by the oil sector are included in the trade figures. Refinery products delivered to Aruban users are registered as import. 
Table A5. Aruba: Changes in Consumer Price Index, 2006-09

(Annual percentage change)

\begin{tabular}{lrrrr}
\hline & 2006 & 2007 & 2008 & 2009 \\
\hline Total index & 3.6 & 5.4 & 9.0 & -2.1 \\
Food and non-alcoholic beverages & 5.2 & 13.7 & 11.2 & 4.6 \\
Alcoholic beverages and tobacco & 0.9 & 11.2 & 3.5 & 4.6 \\
Clothing and footwear & 1.2 & 4.4 & 7.1 & 0.2 \\
Housing & 7.4 & 5.5 & 13.8 & -8.2 \\
Household operation & 1.5 & 4.6 & 3.7 & 2.6 \\
Health & 0.0 & -2.7 & 13.1 & 2.0 \\
Transport & 1.7 & 7.1 & 11.3 & -6.5 \\
Communications & 0.0 & 2.3 & -0.3 & -0.9 \\
Recreation and culture & 3.1 & 8.7 & 4.4 & 2.4 \\
Education & 3.6 & 6.0 & 8.0 & -4.4 \\
Restaurants and hotels & 3.0 & 6.4 & 2.3 & 7.3 \\
Miscellaneous goods and services & 2.1 & -0.2 & 3.5 & 2.8 \\
& & & & \\
Aruba & 3.6 & 5.4 & 9.0 & -2.1 \\
Aruba (excl. energy-related components) & 1.9 & 4.2 & 4.7 & 2.3 \\
Aruba (excl. energy- \& food-related components) & 1.4 & 3.0 & 3.3 & 1.8 \\
United States & 3.2 & 2.9 & 3.8 & -0.4 \\
Curacao & 3.1 & 3.0 & 6.9 & 1.8 \\
The Netherlands & 1.2 & 1.6 & 2.5 & 1.2 \\
& & & & \\
Real exchange rate index (1995=100) 1/ & 105.4 & 108.0 & 115.3 & 111.3 \\
\hline
\end{tabular}

Sources: CBA; CBS Aruba; CBS Netherlands; CBS Netherlands Antilles; Bureau of Labor Statistics.

1/ Relative to the U.S.A. Based on CPI 12-month averages.

Table A6. Aruba: Legal Minimum Wages, 2006-09

(Aruban florins per month)

\begin{tabular}{lrrrr}
\hline & 2006 & 2007 & 2008 & 2009 \\
\hline Category & & & & \\
Construction and industry & 1,360 & 1,460 & 1,505 & 1,543 \\
Household personnel & 635 & 681 & 702 & 720 \\
\hline
\end{tabular}

Source: Aruban Department of Labor. 
Table A7. Aruba: Operations of the Central Government, 2006-09 (Millions of Aruban florins)

\begin{tabular}{|c|c|c|c|c|}
\hline & 2006 & 2007 & 2008 & 2009 \\
\hline Total revenue & 985.2 & $1,034.0$ & $1,365.1$ & $1,108.9$ \\
\hline Tax revenue & 850.2 & 882.2 & 977.0 & 928.9 \\
\hline Income and profit & 397.1 & 313.1 & 353.8 & 338.8 \\
\hline Commodities & 276.4 & 280.7 & 272.7 & 248.7 \\
\hline Property & 54.0 & 58.6 & 68.1 & 66.8 \\
\hline Services & 60.8 & 60.1 & 62.9 & 63.0 \\
\hline Turnover (B.B.O.) & 0.0 & 132.2 & 169.0 & 171.4 \\
\hline Foreign exchange & 62.0 & 37.5 & 50.4 & 40.2 \\
\hline Non tax revenue & 135.0 & 151.8 & 388.1 & 180.0 \\
\hline Grants & 32.7 & 34.1 & 275.5 & 52.4 \\
\hline Other & 102.3 & 117.7 & 112.7 & 127.6 \\
\hline Total expenditure & 924.9 & $1,029.3$ & $1,066.7$ & $1,149.3$ \\
\hline Current expenditure & 851.3 & 950.5 & 965.8 & $1,024.1$ \\
\hline Wage related costs & 533.8 & 527.0 & 542.1 & 570.7 \\
\hline Wages & 309.2 & 300.9 & 304.5 & 315.0 \\
\hline Employers contributions & 84.0 & 83.7 & 89.2 & 101.9 \\
\hline Wage subsidies & 140.6 & 142.4 & 148.5 & 153.8 \\
\hline Goods and services & 158.5 & 181.8 & 189.9 & 203.7 \\
\hline Transfer to AZV & 85.8 & 119.0 & 124.1 & 131.2 \\
\hline Other & 73.2 & 122.7 & 109.7 & 118.5 \\
\hline Subsidies & 26.3 & 23.6 & 23.7 & 25.8 \\
\hline Transfers & 59.7 & 62.2 & 39.1 & 72.4 \\
\hline Contributions/settlements & -12.8 & 36.9 & 46.9 & 20.4 \\
\hline Capital expenditure & 66.5 & 57.5 & 77.8 & 82.3 \\
\hline Investment & 32.0 & 18.9 & 41.6 & 41.2 \\
\hline Development fund spending & 34.5 & 38.7 & 36.2 & 41.1 \\
\hline Lending & 7.1 & 21.3 & 23.1 & 42.9 \\
\hline Lending & 17.2 & 23.7 & 25.5 & 45.3 \\
\hline Repayments & -10.1 & -2.4 & -2.4 & -2.4 \\
\hline Primary balance & 60.4 & 4.7 & 298.4 & -40.4 \\
\hline Interest & 103.9 & 113.2 & 114.9 & 119.1 \\
\hline Domestic & 53.7 & 61.4 & 61.3 & 61.8 \\
\hline External & 50.2 & 51.8 & 53.6 & 57.3 \\
\hline Overall balance & -43.5 & -108.5 & 183.5 & -159.5 \\
\hline Financing & 43.5 & 108.5 & -183.5 & 159.5 \\
\hline Domestic & 68.2 & 64.9 & -102.2 & 145.5 \\
\hline Banks & 4.6 & 50.7 & -0.1 & 63.4 \\
\hline Loans & 23.1 & 23.3 & -11.5 & 58.0 \\
\hline Disbursed & 64.0 & 25.1 & 33.1 & 87.5 \\
\hline Repaid & -40.9 & -1.8 & -44.6 & -29.5 \\
\hline Deposits & -18.5 & 27.4 & 11.4 & 5.4 \\
\hline Non-Bank & 83.7 & 49.3 & 25.9 & 91.0 \\
\hline Disbursed & 105.3 & 55.6 & 44.1 & 149.5 \\
\hline Repaid & -21.6 & -6.3 & -18.2 & -58.5 \\
\hline Central bank & -20.1 & -35.1 & -128.0 & -8.9 \\
\hline Loans & 0.0 & 0.0 & 0.0 & 0.0 \\
\hline Deposits & -20.1 & -35.1 & -128.0 & -8.9 \\
\hline Foreign & 51.5 & 6.6 & 50.7 & 17.6 \\
\hline Disbursed & 92.9 & 82.0 & 135.8 & 60.9 \\
\hline Repaid & -41.4 & -75.4 & -85.1 & -43.3 \\
\hline Unmet financing requirements (arrears) & -76.2 & 37.0 & -132.0 & -3.6 \\
\hline \multicolumn{5}{|l|}{ Memorandum items: } \\
\hline Gross debt & 2,012 & 2,140 & 2,050 & 2,180 \\
\hline Domestic debt & 1,078 & 1,158 & 1,005 & 1,113 \\
\hline Foreign debt & 934 & 982 & 1,045 & 1,066 \\
\hline Gross assets (govt. deposits) & 188 & 195 & 297 & 298 \\
\hline Net debt & 1,824 & 1,945 & 1,753 & 1,882 \\
\hline
\end{tabular}

Sources: Aruban authorities; and IMF staff estimates. 
Table A8. Aruba: Operational Budget of the Social Insurance Bank (SVB), 2005-09

\begin{tabular}{|c|c|c|c|c|c|}
\hline & 2005 & 2006 & 2007 & 2008 & 2009 \\
\hline \multicolumn{6}{|c|}{ (Millions of Aruban florins) } \\
\hline Total contributions & 186.4 & 187.5 & 203.8 & 226.9 & 222.3 \\
\hline Total benefits & 201.4 & 199.9 & 202.8 & 223.4 & 233.6 \\
\hline Administrative and interest costs & -10.9 & -10.3 & -10.2 & -10.8 & 0.2 \\
\hline Administrative costs & -11.3 & -11.0 & -10.7 & -11.6 & -0.6 \\
\hline Interest costs & 0.4 & 0.7 & 0.5 & 0.7 & 0.8 \\
\hline Overall balance & -26.0 & -22.7 & -9.3 & -7.4 & -11.0 \\
\hline \multicolumn{6}{|l|}{ Old age and widowers and orphans insurance } \\
\hline Contributions & 139.8 & 165.2 & 171.8 & 192.1 & 187.5 \\
\hline Benefits & 171.1 & 175.0 & 179.7 & 198.7 & 205.4 \\
\hline Administrative and interest costs & 1.8 & 2.2 & 2.2 & 1.9 & -0.5 \\
\hline Administrative costs & -2.2 & -2.0 & -2.1 & -2.3 & -0.5 \\
\hline Interest costs & 3.9 & 4.2 & 4.3 & 4.1 & 0.0 \\
\hline Balance & -29.5 & -7.6 & -5.6 & -4.7 & -18.4 \\
\hline \multicolumn{6}{|l|}{ Sickness and accident insurance } \\
\hline Contributions & 44.8 & 21.5 & 30.4 & 33.3 & 33.3 \\
\hline Benefits & 29.5 & 24.4 & 23.0 & 23.9 & 25.8 \\
\hline Administrative and interest costs & -12.9 & -12.8 & -12.7 & -13.3 & -0.1 \\
\hline Administrative costs & -8.8 & -8.6 & -8.2 & -8.9 & -0.1 \\
\hline Interest costs & -4.1 & -4.3 & -4.5 & -4.4 & 0.0 \\
\hline Balance & 2.4 & -15.7 & -5.3 & -3.9 & 7.3 \\
\hline \multicolumn{6}{|l|}{ Severance insurance } \\
\hline Contributions & 1.8 & 0.8 & 1.5 & 1.5 & 1.5 \\
\hline Interest revenues & 0.5 & 0.7 & 0.7 & 1.1 & 0.8 \\
\hline Benefits & 0.8 & 0.4 & 0.1 & 0.9 & 2.3 \\
\hline Administrative costs & -0.3 & -0.4 & -0.4 & -0.4 & \\
\hline Balance & 1.1 & 0.6 & 1.6 & 1.2 & 0.0 \\
\hline \multicolumn{6}{|c|}{ (Overall balances, percent of GDP) } \\
\hline Total contributions & 4.5 & 4.3 & 4.4 & 4.5 & 4.8 \\
\hline Total benefits & 4.8 & 4.6 & 4.4 & 4.5 & 5.1 \\
\hline Administrative and interest costs & -0.3 & -0.2 & -0.2 & -0.2 & 0.0 \\
\hline Administrative costs & -0.3 & -0.3 & -0.2 & -0.2 & 0.0 \\
\hline Interest costs & 0.0 & 0.0 & 0.0 & 0.0 & 0.0 \\
\hline Overall balance & -0.6 & -0.5 & -0.2 & -0.1 & -0.2 \\
\hline \multicolumn{6}{|l|}{ Old age and widowers and orphans insurance } \\
\hline Contributions & 3.4 & 3.8 & 3.7 & 3.8 & 4.1 \\
\hline Benefits & 4.1 & 4.0 & 3.9 & 4.0 & 4.5 \\
\hline Administrative and interest costs & 0.0 & 0.1 & 0.0 & 0.0 & 0.0 \\
\hline Administrative costs & -0.1 & 0.0 & 0.0 & 0.0 & 0.0 \\
\hline Interest costs & 0.1 & 0.1 & 0.1 & 0.1 & 0.0 \\
\hline Balance & -0.7 & -0.2 & -0.1 & -0.1 & -0.4 \\
\hline \multicolumn{6}{|l|}{ Sickness and accident insurance } \\
\hline Contributions & 1.1 & 0.5 & 0.7 & 0.7 & 0.7 \\
\hline Benefits & 0.7 & 0.6 & 0.5 & 0.5 & 0.6 \\
\hline Administrative and interest costs & -0.3 & -0.3 & -0.3 & -0.3 & 0.0 \\
\hline Administrative costs & -0.2 & -0.2 & -0.2 & -0.2 & 0.0 \\
\hline Interest costs & -0.1 & -0.1 & -0.1 & -0.1 & 0.0 \\
\hline Balance & 0.1 & -0.4 & -0.1 & -0.1 & 0.2 \\
\hline \multicolumn{6}{|l|}{ Severance insurance } \\
\hline Contributions & 0.04 & 0.02 & 0.03 & 0.03 & 0.03 \\
\hline Interest revenues & 0.01 & 0.02 & 0.02 & 0.02 & 0.02 \\
\hline Benefits & 0.02 & 0.01 & 0.00 & 0.02 & 0.05 \\
\hline Administrative costs & -0.01 & -0.01 & -0.01 & -0.01 & \\
\hline Balance & 0.03 & 0.01 & 0.04 & 0.02 & 0.00 \\
\hline \multicolumn{6}{|c|}{ Memorandum items: } \\
\hline \multicolumn{6}{|l|}{ Number of beneficiaries per category } \\
\hline Old age and widowers and orphans insurance & 27,367 & 15,193 & 16,355 & 17,113 & 17,880 \\
\hline Old age & 14,208 & 14,112 & 14,582 & 15,312 & 16,081 \\
\hline Widowers and orphans & 13,159 & 1,081 & 1,773 & 1,801 & 1,799 \\
\hline Sickness and accident insurance & $\ldots$ & 33,901 & 31,682 & 35,820 & 35,321 \\
\hline Sickness & $\ldots$ & 31,372 & 29,414 & 33,440 & 33,589 \\
\hline Accident & $\ldots$ & 2,529 & 2,268 & 2,380 & 1,732 \\
\hline Severance insurance & $\ldots$ & 121 & 47 & 187 & 414 \\
\hline Number of insured per category & & & & & \\
\hline Old age and widowers and & & & & & \\
\hline orphans insurance & $\ldots$ & 137,146 & 138,206 & 140,242 & 141,766 \\
\hline Old age & $\ldots$ & 68,573 & 69,103 & 70,121 & 70,883 \\
\hline Widowers and orphans & $\ldots$ & 68,573 & 69,103 & 70,121 & 70,883 \\
\hline Sickness and accident insurance & $\ldots$ & 65,821 & 67,376 & 69,893 & 67,176 \\
\hline Sickness & $\ldots$ & 30,048 & 30,589 & 31,917 & 30,716 \\
\hline Accident & $\ldots$ & 35,773 & 36,787 & 37,976 & 36,460 \\
\hline Severance insurance 1/ & $\ldots$ & 35,773 & 36,787 & 37,976 & 36,460 \\
\hline
\end{tabular}

Source: SVB.

1/ Concerns the same category of insured as those insured for accidents. 
Table A9. Aruba: Financial Balance of AZV, 2006-09 (Percent of GDP)

\begin{tabular}{lcccc}
\hline & 2006 & 2007 & 2008 & 2009 \\
\hline Revenue & 4.1 & 3.6 & 3.6 & 4.0 \\
Expenditures & 6.3 & 6.4 & 6.4 & 7.2 \\
$\quad$ Health & 5.9 & 6.0 & 6.0 & 6.8 \\
Management & 0.3 & 0.4 & 0.4 & 0.4 \\
Other Costs & 0.0 & 0.1 & 0.0 & 0.0 \\
Deficit & -2.2 & -2.8 & -2.8 & -3.2 \\
Transfer from Central Govt & 2.2 & 2.8 & 2.8 & 3.2 \\
Primary Transfer & 2.2 & 2.1 & 2.4 & 2.7 \\
Additional Transfer & 0.0 & 0.7 & 0.4 & 0.5 \\
\hline
\end{tabular}

Sources: Aruban authorities; and IMF staff estimates.

Table A10. Aruba: Balance of Payments Summary, 2005-09 (Millions of Aruban florins)

\begin{tabular}{lrrrrr}
\hline & 2005 & 2006 & 2007 & 2008 & 2009 \\
\hline Current account & -354 & -63 & -178 & -272 & 283 \\
Merchandise trade balance & 38 & -213 & -290 & -887 & -847 \\
Exports of goods & 6,235 & 6,564 & 4,817 & 6,632 & 2,588 \\
$\quad$ Imports of goods & 6,197 & 6,777 & 5,107 & 7,520 & 3,434 \\
Services & 701 & 580 & 1,018 & 1,009 & 1,387 \\
$\quad$ Exports of services & 2,334 & 2,373 & 2,709 & 3,029 & 2,866 \\
$\quad$ Imports of services & 1,633 & 1,792 & 1,691 & 2,019 & 1,479 \\
$\quad$ Of which: Travel and tourism & 1,606 & 1,553 & 1,778 & 1,988 & 1,800 \\
Income and current transfers & $-1,093$ & -430 & -906 & -394 & -257 \\
Income & -867 & -209 & -723 & -190 & -122 \\
Current transfers & -226 & -221 & -183 & -204 & -136 \\
Financial and capital account & 301 & 163 & 244 & 696 & -200 \\
Financial account & 268 & 125 & 211 & 415 & -261 \\
$\quad$ Direct investment & 214 & 1,039 & -224 & 345 & 142 \\
Portfolio investment (including banking transactions) & 6 & -86 & 106 & 113 & 10 \\
Loans to general government & 58 & 83 & 53 & 94 & 42 \\
Banking sector (net increase in liabilities) & -56 & -88 & -51 & -113 & -43 \\
Other & 46 & -823 & 327 & -24 & -411 \\
Capital account & 33 & 38 & 33 & 280 & 61 \\
Errors and omissions & 12 & 0 & 11 & -21 & -20 \\
Change in reserves (-=increase) 1/ & 40 & -99 & -77 & -403 & -61 \\
\hline
\end{tabular}

Sources: Central Bank of Aruba; and staff estimates.

$1 /$ Including gold, excluding revaluation differences. 
Table A11. Aruba: Balance of Payments Summary, 2005-09

(Percent of GDP)

\begin{tabular}{lrrrrr}
\hline & 2005 & 2006 & 2007 & 2008 & 2009 \\
\hline Current account & -8.5 & -1.5 & -3.8 & -5.4 & 6.1 \\
Merchandise trade balance & 0.9 & -4.9 & -6.2 & -17.8 & -18.4 \\
Exports of goods & 149.9 & 151.5 & 103.4 & 132.7 & 56.1 \\
Imports of goods & 149.0 & 156.4 & 109.6 & 150.5 & 74.5 \\
Services & 16.9 & 13.4 & 21.9 & 20.2 & 30.1 \\
Exports of services & 56.1 & 54.7 & 58.2 & 60.6 & 62.1 \\
Imports of services & 39.3 & 41.4 & 36.3 & 40.4 & 32.1 \\
Of which: Travel and tourism & 38.6 & 35.8 & 38.2 & 39.8 & 39.0 \\
Income and current transfers & -26.3 & -9.9 & -19.5 & -7.9 & -5.6 \\
Income & -20.8 & -4.8 & -15.5 & -3.8 & -2.6 \\
Current transfers & -5.4 & -5.1 & -3.9 & -4.1 & -2.9 \\
Financial and capital account & 7.2 & 3.8 & 5.2 & 13.9 & -4.3 \\
Financial account & 6.4 & 2.9 & 4.5 & 8.3 & -5.7 \\
Direct investment & 5.1 & 24.0 & -4.8 & 6.9 & 3.1 \\
Portfolio investment & 0.1 & -2.0 & 2.3 & 2.3 & 0.2 \\
Loans to general government & 1.4 & 1.9 & 1.1 & 1.9 & 0.9 \\
Banking sector (net increase in liabilities) & -1.3 & -2.0 & -1.1 & -2.3 & -0.9 \\
Other & 1.1 & -19.0 & 7.0 & -0.5 & -8.9 \\
Capital account & 0.8 & 0.9 & 0.7 & 5.6 & 1.3 \\
Errors and omissions & 0.3 & 0.0 & 0.2 & -0.4 & -0.4 \\
Change in reserves (--increase) 1/ & 1.0 & -2.3 & -1.7 & -8.1 & -1.3 \\
\hline
\end{tabular}

Sources: Central Bank of Aruba; and staff estimates.

1 / Including gold, excluding revaluation differences. 
Table A12. Aruba: Non-Oil Balance of Payments Summary, 2005-09 (Millions of Aruban florins)

\begin{tabular}{lrrrrr}
\hline & 2005 & 2006 & 2007 & 2008 & 2009 \\
\hline Current account & -357 & -641 & -263 & -154 & 37 \\
Merchandise trade balance & $-1,335$ & $-1,415$ & $-1,407$ & $-1,478$ & $-1,335$ \\
Exports of goods & 25 & 26 & 18 & 27 & 22 \\
Imports of goods & 1,360 & 1,441 & 1,425 & 1,505 & 1,357 \\
Services & 1,293 & 1,148 & 1,469 & 1,650 & 1,604 \\
Exports of services & 2,325 & 2,365 & 2,704 & 3,021 & 2,859 \\
Imports of services & 1,032 & 1,217 & 1,235 & 1,370 & 1,256 \\
Of which: Travel and tourism (net) & 1,598 & 1,548 & 1,774 & 1,983 & 1,794 \\
Income and current transfers & -315 & -374 & -325 & -326 & -232 \\
Income & -158 & -212 & -182 & -189 & -120 \\
Current transfers & -157 & -162 & -142 & -137 & -112 \\
Financial and capital account & 305 & 740 & 330 & 580 & 44 \\
Financial account & 272 & 702 & 297 & 300 & -17 \\
$\quad$ Direct investment & 222 & 533 & 285 & 334 & 139 \\
Portfolio investment & -13 & -79 & 78 & 112 & 2 \\
Loans to general government & 55 & 83 & 53 & 94 & 42 \\
Banking sector (net increase in liabilities) & -134 & 76 & -361 & -420 & -341 \\
Other & 143 & 89 & 243 & 181 & 142 \\
Capital account & 33 & 38 & 33 & 280 & 61 \\
Errors and omissions & 13 & -1 & 10 & -23 & -19 \\
Change in reserves (-=increase) 1/ & 40 & -99 & -77 & -403 & -61 \\
\hline
\end{tabular}

Sources: Central Bank of Aruba; and staff estimates.

$1 /$ Including gold, excluding revaluation differences.

Table A13. Aruba: Non-Oil Balance of Payments Summary, 2005-09 (Percent of GDP)

\begin{tabular}{lrrrrr}
\hline & 2005 & 2006 & 2007 & 2008 & 2009 \\
\hline Current Account & -8.6 & -14.8 & -5.6 & -3.1 & 0.8 \\
Merchandise trade balance & -32.1 & -32.6 & -30.2 & -29.6 & -29.0 \\
Exports of goods & 0.6 & 0.6 & 0.4 & 0.5 & 0.5 \\
Imports of goods & 32.7 & 33.2 & 30.6 & 30.1 & 29.4 \\
Services & 31.1 & 26.5 & 31.5 & 33.0 & 34.8 \\
Exports of services & 55.9 & 54.6 & 58.0 & 60.5 & 62.0 \\
Imports of services & 24.8 & 28.1 & 26.5 & 27.4 & 27.2 \\
Of which: Travel and tourism (net) & 38.4 & 35.7 & 38.1 & 39.7 & 38.9 \\
Income and current transfers & -7.6 & -8.6 & -7.0 & -6.5 & -5.0 \\
Income & -3.8 & -4.9 & -3.9 & -3.8 & -2.6 \\
Current transfers & -3.8 & -3.7 & -3.1 & -2.7 & -2.4 \\
Financial and capital account & 7.3 & 17.1 & 7.1 & 11.6 & 0.9 \\
Financial account & 6.5 & 16.2 & 6.4 & 6.0 & -0.4 \\
$\quad$ Direct investment & 5.3 & 12.3 & 6.1 & 6.7 & 3.0 \\
Portfolio investment & -0.3 & -1.8 & 1.7 & 2.2 & 0.0 \\
Loans to general government & 1.3 & 1.9 & 1.1 & 1.9 & 0.9 \\
Banking sector (net increase in liabilities) & -3.2 & 1.8 & -7.8 & -8.4 & -7.4 \\
Other & 3.4 & 2.0 & 5.2 & 3.6 & 3.1 \\
Capital account & 0.8 & 0.9 & 0.7 & 5.6 & 1.3 \\
Errors and omissions & 0.3 & 0.0 & 0.2 & -0.5 & -0.4 \\
Change in reserves (-=increase) 1/ & 1.0 & -2.3 & -1.7 & -8.1 & -1.3 \\
\hline
\end{tabular}

Sources: Central Bank of Aruba; and staff estimates.

$1 /$ Including gold, excluding revaluation differences. 
Table A14. Aruba: Bank-Like Institutions-Balance Sheet, 2005-09

(Millions of Aruban florins, end of period)

\begin{tabular}{lrrrrr}
\hline & 2005 & 2006 & 2007 & 2008 & $20091 /$ \\
\hline Assets & & & & & \\
$\quad$ Cash and due from banks & 64.3 & 48.5 & 33.8 & 43.7 & 58.2 \\
$\quad$ Investments & 12.0 & 23.7 & 28.0 & 25.6 & 24.9 \\
Loans & 413.2 & 420.0 & 474.4 & 495.5 & 486.4 \\
$\quad$ Commercial 2/ & 117.1 & 103.9 & 144.5 & 167.1 & 162.2 \\
$\quad$ Individuals 3/ & 296.1 & 316.1 & 329.9 & 328.4 & 324.2 \\
$\quad$ Government & 0.0 & 0.0 & 0.0 & 0.0 & 0 \\
Other assets & 114.4 & 108.4 & 119.9 & 124.5 & 137 \\
Total assets & 603.9 & 600.6 & 656.1 & 689.3 & 706.5 \\
Capital and liabilities & & & & & \\
Deposits & 0.0 & 0.0 & 0.0 & 0.0 & 0 \\
Borrowings & 323.2 & 354.5 & 385.8 & 404.8 & 401.2 \\
$\quad$ Commercial & 271.6 & 296.9 & 321.2 & 328.7 & 322.7 \\
$\quad$ Individuals & 0.0 & 0.0 & 0.0 & 6.5 & 6.9 \\
$\quad$ Government & 51.6 & 57.6 & 64.6 & 69.6 & 71.6 \\
$\quad$ Other liabilities & 73.9 & 35.7 & 34.8 & 35.0 & 36.6 \\
Capital and reserves 4/ & 206.8 & 210.4 & 235.5 & 249.5 & 268.7 \\
Total capital and liabilities & 603.9 & 600.6 & 656.1 & 689.3 & 706.5 \\
\hline
\end{tabular}

Source: Central Bank of Aruba.

1/ Preliminary figures

2/ Corrected for loan loss provisions.

$3 /$ Corrected for unearned income.

4/ Including general (unallocated) reserves.

Table A 15: Aruba: Life Insurance Companies-Balance Sheet, 2005-08 (Millions of Aruban florins, end of period)

\begin{tabular}{lrrrr}
\hline & 2005 & 2006 & 2007 & 2008 \\
\hline Assets & & & & \\
$\quad$ Investments & 373.2 & 397.9 & 456.8 & 489.7 \\
$\quad$ Shares & 2.6 & 2.9 & 2.8 & 2.1 \\
Bonds & 190.1 & 219.5 & 247.6 & 250.1 \\
Real estate & 14.0 & 10.3 & 10.3 & 10.1 \\
Time deposits & 53.7 & 64.8 & 72.6 & 93.7 \\
$\quad$ Mortgage loans & 72.9 & 59.1 & 72.6 & 89.7 \\
$\quad$ Other loans & 39.9 & 41.3 & 50.9 & 44.0 \\
Fixed assets & 0.4 & 7.3 & 8.2 & 5.8 \\
Due from affiliated companies & 19.4 & 22.5 & 20.0 & 22.3 \\
Current assets & 58.5 & 64.5 & 65.1 & 100.2 \\
Total assets & 451.5 & 492.2 & 550.1 & 618.0 \\
Capital and liabilities & & & & \\
Technical provisions & 364.6 & 400.9 & 443.0 & 499.1 \\
Long-term liabilities & 0.0 & 0.0 & 0.8 & 0.2 \\
Due to affiliated companies & 5.0 & 5.4 & 6.5 & 16.8 \\
Current liabilities & 22.1 & 19.0 & 24.7 & 32.7 \\
Capital and reserves & 59.8 & 66.9 & 75.1 & 69.2 \\
Total capital and liabilities & 451.5 & 492.2 & 550.1 & 618.0 \\
\hline
\end{tabular}

Source: Central Bank of Aruba. 
Table A 16: Aruba: Pension Funds-Balance Sheet, 2005-09

(Millions of Aruban florins, end of period)

\begin{tabular}{lrrrrr}
\hline & 2005 & 2006 & 2007 & 2008 & $2009^{1 /}$ \\
\hline Assets & 192 & 221 & 240 & 197 & 221 \\
Investments & 52 & 56 & 63 & 50 & 55 \\
$\quad$ Shares & 58 & 72 & 86 & 78 & 90 \\
$\quad$ Bonds & 10 & 10 & 10 & 10 & 10 \\
Real estate & 36 & 41 & 36 & 15 & 18 \\
$\quad$ Time deposits & 14 & 15 & 16 & 16 & 18 \\
$\quad$ Mortgage loans & 23 & 28 & 29 & 29 & 30 \\
$\quad$ Other investments & 0 & 0 & 0 & 0 & 0 \\
Fixed assets & 21 & 14 & 15 & 16 & 20 \\
$\quad$ Current assets & 213 & 235 & 255 & 213 & 241 \\
Total assets & & & & & \\
Capital and liabilities & 182 & 195 & 216 & 196 & 209 \\
$\quad$ Technical provisions & 0 & 0 & 0 & 0 & 0 \\
Long-term liabilities & 2 & 2 & 2 & 2 & 2 \\
$\quad$ Current liabilities & 30 & 37 & 36 & 16 & 30 \\
$\quad$ Capital and reserves & 213 & 235 & 255 & 213 & 241 \\
Total capital and liabilities & & & &
\end{tabular}

Source: Central Bank of Aruba.

1/ Preliminary figures

Table A17. Aruba: Construction Indicators, 2005-09

\begin{tabular}{lrrrrr}
\hline & 2005 & 2006 & 2007 & 2008 & 2009 \\
\hline Number of construction permits granted & 1,584 & 1,303 & 1,151 & 962 & 857 \\
Houses & 1,092 & 782 & 674 & 525 & 499 \\
Apartments & 25 & 30 & 28 & 39 & 33 \\
Office buildings & 15 & 22 & 25 & 13 & 9 \\
Stores and shopping malls & 25 & 34 & 29 & 16 & 11 \\
Others & 427 & 435 & 395 & 369 & 305 \\
Total value of construction permits (Afl. million) & 283.2 & 525.7 & 447.6 & 670.5 & 343.2 \\
Houses & 113.7 & 108.6 & 115.3 & 105.3 & 93.6 \\
Apartments & 5.0 & 13.8 & 12.4 & 62.7 & 13.2 \\
Office buildings & 10.1 & 39.2 & 40.9 & 11.3 & 8.1 \\
Stores and shopping malls & 17.4 & 78.2 & 64.4 & 29.5 & 10.2 \\
Others & 137.0 & 286.0 & 214.5 & 461.7 & 218.1 \\
Total cement imported (x 1,000 Kg) & 88,719 & 78,413 & 77,832 & 67,040 & 49,746 \\
Number of electrical installations approved & 2,763 & 2,463 & 2,138 & 2,272 & 2,021 \\
Houses & 857 & 686 & 560 & 583 & 467 \\
Apartments & 258 & 204 & 259 & 277 & 199 \\
Enterprises & 296 & 252 & 291 & 292 & 231 \\
Others & 1,352 & 1,321 & 1,028 & 1,120 & 1,124 \\
\hline
\end{tabular}

Sources: Department of Public Works; Department of Technical Inspection; CBS. 
Table A18. Aruba: Utilities, 2005-09

\begin{tabular}{lrrrrr}
\hline & 2005 & 2006 & 2007 & 2008 & 2009 \\
\hline Water & & & & & \\
Quantity (x 1,000 m3) & 11,399 & 11,474 & 11,750 & 11,445 & 11,383 \\
Value (Afl. million) & 82.9 & 95.9 & 102.3 & 122.9 & 103.3 \\
Connected premises & 34,905 & 35,989 & 36,824 & 37,992 & 38,857 \\
Electricity & & & & & \\
Quantity (x 1,000 KWH) & 759,336 & 761,362 & 781,073 & 764,291 & 773,909 \\
Value (Afl. million) & 228.2 & 262.8 & 279.3 & 344.1 & 283.4 \\
Connections & 40,765 & 41,502 & 42,419 & 43,062 & 43,635 \\
Number of users & 36,737 & 37,630 & 38,495 & 39,207 & 39,743 \\
Gas & & & & & \\
$\quad$ Quantity (x 1,000 pounds) & 19,133 & 19,275 & 19,979 & 20,486 & 20,511 \\
Value (Afl. million) & 11.8 & 12.6 & 15.0 & 18.3 & 20.5 \\
Households & 7,416 & 7,387 & 7,355 & 7,496 & 7,654 \\
Commercial users & 11,717 & 11,888 & 12,624 & 12,990 & 12,857 \\
Utilities index & 138.9 & 139.4 & 142.9 & 140.1 & 141.1 \\
\hline
\end{tabular}

Sources: WEB Aruba N.V.; N.V. ELMAR; Arugas N.V. 
Table A19. Aruba: Housing Mortgages, 1996-09

(Millions of Aruban florins, end of period)

\begin{tabular}{rrrrrrr}
\hline & $\begin{array}{r}\text { Commercial } \\
\text { banks } \\
(1)\end{array}$ & $\begin{array}{r}\text { Mortgage } \\
\text { banks } \\
(2)\end{array}$ & $\begin{array}{r}\text { Pension } \\
\text { funds } \\
(3)\end{array}$ & $\begin{array}{r}\text { Life insurance } \\
\text { companies }\end{array}$ & Other & Total \\
& 239 & 195 & 36 & 34 & 5 & (4) \\
\hline 1996 & 259 & 239 & 41 & 40 & 5 & (6) \\
1997 & 296 & 269 & 50 & 49 & 5 & 670 \\
1998 & 345 & 277 & 59 & 61 & 6 & 748 \\
1999 & 395 & 283 & 63 & 73 & 8 & 822 \\
2000 & 438 & 296 & 74 & 72 & 9 & 889 \\
2001 & 485 & 303 & 84 & 68 & 4 & 944 \\
2002 & 544 & 281 & 91 & 62 & 18 & 996 \\
2003 & 581 & 287 & 113 & 57 & 17 & 1056 \\
2004 & 704 & 229 & 126 & 54 & 16 & 1129 \\
2005 & 747 & 243 & 138 & 59 & 15 & 1202 \\
2006 & 786 & 245 & 156 & 72 & 18 & 1276 \\
2007 & 797 & 240 & 184 & 89 & 19 & 1330 \\
2008 & 819 & 232 & 208 & 91 & 19 & 1368 \\
2009 & & & & & & \\
\hline
\end{tabular}

Source: Central Bank of Aruba. 